\title{
Effective-Range-Expansion Study of Near Threshold Heavy-Flavor Resonances
}

\author{
Rui Gao, ${ }^{1}$ Zhi-Hui Guo $\mathbb{D D}^{1},{ }^{1}$ Xian-Wei Kang, ${ }^{2}$ and J. A. Oller ${ }^{3}$ \\ ${ }^{1}$ Department of Physics and Hebei Advanced Thin Films Laboratory, Hebei Normal University, Shijiazhuang 050024, China \\ ${ }^{2}$ College of Nuclear Science and Technology, Beijing Normal University, Beijing 100875, China \\ ${ }^{3}$ Departamento de Física, Universidad de Murcia, E-30071 Murcia, Spain
}

Correspondence should be addressed to Zhi-Hui Guo; zhguo@hebtu.edu.cn

Received 26 December 2018; Revised 5 April 2019; Accepted 15 May 2019; Published 16 June 2019

Academic Editor: Burak Bilki

Copyright (C) 2019 Rui Gao et al. This is an open access article distributed under the Creative Commons Attribution License, which permits unrestricted use, distribution, and reproduction in any medium, provided the original work is properly cited. The publication of this article was funded by SCOAP ${ }^{3}$.

In this work, we study the resonances near the thresholds of the open heavy-flavor hadrons using the effective-range-expansion method. The unitarity, analyticity, and compositeness coefficient are also taken into account in our theoretical formalism. We consider the $Z_{c}(3900), X(4020), \chi_{c 1}(4140), \psi(4260)$, and $\psi(4660)$. The scattering lengths and effective ranges from the relevant elastic $S$-wave scattering amplitudes are determined. Tentative discussions on the inner structures of the aforementioned resonances are given.

\section{Introduction}

Since the discovery of the $X(3872)$ [1], the study of the exotic hidden heavy-flavor hadrons has become one of the most important and active research topics in particle physics. Up to now more than twenty of the so-called $X Y Z$ hadrons are observed by experiments [2] and we refer to $[3,4]$ for recent comprehensive reviews on this subject. One of the most important features of the newly observed hadrons is that they are usually close to the nearby thresholds of the open heavy-flavor states. As a result typically one needs to properly take into account the threshold effects when studying those possible exotic states. The effective range expansion (ERE), which is based on the three-momentum expansion near the threshold, provides a useful tool to address the dynamics in the energy region around the relevant threshold in question $[5,6]$.

By combining the ERE, unitarity, analyticity, and the compositeness coefficients developed in $[7,8]$, we have successfully analyzed several non-ordinary hadronic states that lie close to the thresholds of the underlying twoparticle states, including the $\Lambda_{c}$ (2595) [9], $Z_{b}(10610)$, and $Z_{b}(10650)$ [10], and the newly observed pentaquark candidates $P_{c}(4312), P_{c}(4440)$ and $P_{c}(4457)$ [11]. The essential idea of the formalism is that we construct the elastic unitarized partial-wave amplitude using the ERE as the kernel, which includes the scattering length $a$ and effective range $r$ as free parameters. The latter are determined by reproducing the values of the mass and width of the observed resonance. We always obtain real values consistently with the assumption of only one-channel scattering. Then the residue of the resonance pole, which corresponds to the coupling strength of the resonance to the interacting two-particle state, can be obtained as well. With all of these ingredients, we can apply the compositeness formalism to infer the probability to find the two-particle state inside the resonance. In this work we first briefly recapitulate the essentials of the theoretical formalism. Then we tentatively generalize this formalism to other newly observed hadronic states, including the $Z_{c}(3900)$ near the $D \bar{D}^{*}+$ c.c. (denoted shortly as $D \bar{D}^{*}$ in the following) threshold, the $X(4020)$ near the $D^{*} \bar{D}^{*}$ threshold, the $\chi_{c 1}(4140)$ near the $D_{s}^{ \pm} D_{s}^{* \mp}$ (denoted as $D_{s} \bar{D}_{s}^{*}$ in the following) threshold, the $\psi(4260)$ near the $D_{1} \bar{D}+$ c.c. (denoted shortly as $D_{1} \bar{D}$ in the following) threshold, and the $\psi(4660)$ near the $\Lambda_{c} \bar{\Lambda}_{c}$ threshold [2]. Historically, there is also a state named $X(4630)$ that we identify with the resonance $\psi(4660)$ as in the PDG [2] and [12-14]. 


\section{Effective Range Expansion and Compositeness Coefficients of Resonances}

The basic staring point of our theoretical formalism is the ERE up to the next-to-leading order:

$$
t(E)=\frac{1}{-1 / a+(1 / 2) r k^{2}-i k},
$$

with $a$ the scattering length, $r$ the effective range, and $k$ the three-momentum in the center of mass (CM) frame. At a given $C M$ energy $E$ around the threshold, one can write the nonrelativistic three-momentum as

$$
k=\sqrt{2 \mu_{m}\left(E-m_{\mathrm{th}}\right)},
$$

where the reduced mass of the system with masses $m_{1}$ and $m_{2}$ is $\mu_{m}=m_{1} m_{2} /\left(m_{1}+m_{2}\right)$ and the threshold is given by $m_{\mathrm{th}}=m_{1}+m_{2}$.

We mention that a more general expression to write the scattering amplitude near threshold is to include the so-called Castillejo-Dalitz-Dyson (CDD) poles. The standard ERE in (1) can be obtained by expanding the full expression with CDD poles $[9,10]$. However when the CDD pole happens to be near the threshold, the expansion in terms of $k^{2}$ will be invalid, or at least quite limited to a tiny region. One of the prominent features in this situation is the huge effective range $r$, which usually becomes much larger than its standard value around $1 \mathrm{fm}$. If this is the case, one has to work explicitly with the CDD pole in the full expression, as done in $[9,10,18]$.

The partial-wave amplitude given in (1) corresponds to the physical one in the first Riemann sheet (RS). Its expression in the second RS is given by

$$
t_{\mathrm{II}}(E)=\frac{1}{-1 / a+(1 / 2) r k^{2}+i k} .
$$

The resonance poles only appear in the second RS. Comparing with (1) and (3), there is a change of sign for the $k$ term. Notice that, in the conventions of (1) and (3), the threemomentum $k$ should be evaluated with $\operatorname{Im} k>0$.

Let us now consider a resonance $R$ whose pole position in the unphysical RS is located at $E=E_{R}$, with $E_{R}=$ $M_{R}-i \Gamma_{R} / 2$. For a conventional narrow-width resonance, one can identify $M_{R}$ and $\Gamma_{R}$ as its mass and width, respectively. The corresponding three-momentum at the resonance pole is then given by

$$
k_{R}=\sqrt{2 \mu_{m}\left(E_{R}-m_{\mathrm{th}}\right)} .
$$

For later convenience, we further define

$$
k_{R}=k_{r}+i k_{i}, \quad k_{i}>0 .
$$

One has to be careful when evaluating $k_{R}$ in terms of the threshold $m_{\text {th }}$ and the resonance parameters $M_{R}$ and $\Gamma_{R}$, specially distinguishing the sign of $M_{R}-m_{\mathrm{th}}$. Detailed discussions can be found in [10].

The resonance pole corresponds to the zero of the denominator of $t_{\mathrm{II}}(E)$; i.e., at the pole position one has

$$
-\frac{1}{a}+\frac{1}{2} r k_{R}^{2}+i k_{R}=0 .
$$

By a straightforward algebraic manipulation, one can solve $a$ and $r$ in terms of $k_{r}$ and $k_{i}$ :

$$
\begin{aligned}
& a=-\frac{2 k_{i}}{\left|k_{R}\right|^{2}}, \\
& r=-\frac{1}{k_{i}} .
\end{aligned}
$$

Substituting (7) and (8) into (3), one can write the partialwave amplitude around the resonance pole $E_{R}$ as

$$
t_{I I}(k)=\frac{-k_{i} / k_{r}}{k-k_{R}}+\cdots,
$$

where the ellipses stand for the neglected terms when expanding the denominator of (3) in terms of $k-k_{R}$. From (9), we can identify the residue at the pole in the variable $k$, which turns out to be

$$
\gamma_{k}^{2}=-\frac{k_{i}}{k_{r}}>0,
$$

since $k_{r}<0$. Alternatively one can also expand the partialwave amplitude around the pole as

$$
t_{I I}(E)=-\frac{\gamma^{2}}{s-E_{R}^{2}}+\cdots .
$$

The residue $\gamma^{2}$ is related to $\gamma_{k}^{2}$ as

$$
\gamma_{k}^{2}=-\left.\gamma^{2} \frac{d k}{d s}\right|_{k_{R}}=-\frac{\mu_{m} \gamma^{2}}{2 E_{R} k_{R}} .
$$

In [7], a probabilistic interpretation for the compositeness $X$ of the two-particle state inside the resonance is derived. The value of $X$ can be calculated once the resonance pole position and the corresponding residues are provided. Around the two-particle threshold, the probability $X$ reduces to the following [10]:

$$
X=\left|\gamma_{k}\right|^{2}
$$

However, we point out that the probabilistic interpretation of $X$ is restricted to resonances with $M_{R}>m_{\text {th }}$ [7]. In [15-17, 19-24], other approaches to generalize the Weinberg's compositeness of bound states [25] to the resonances are discussed. We compare below our results with some of them in Section 3.

In the next section, we proceed to study several nearthreshold resonances within the present ERE approach. Let us notice that if this type of ERE study is applied to a near-threshold resonance which is composite of the nearby channel (the so-called elastic one) then $X \simeq 1$. From here it also follows that if we apply this type of ERE study to a nearthreshold resonance and it results that $X \ll 1$, then one can conclude for sure that this resonance is not a composite one of the elastic channel. On the other hand, if it results that $X \simeq 1$ then the interpretation of this resonance as a composite one of the elastic channel is favored (In a similar sense that a cloudy sky favors rainfall.). 
TABLE 1: In the second and third columns, the masses and widths of the $Z_{c}(3900), X(4020), \psi(4260)$ and $\psi(4660)$ from the PDG are given. For the $\chi_{c 1}(4140)$, we have distinguished three different determinations: LHCb, the average without LHCb (LHCb) and the PDG average. To make a conservative error estimate, the largest error bars are taken for the asymmetric ones in the values from the LHCb and PDG. We assume that the $S$-wave two-particle states shown in the fourth column are responsible for the resonance poles. The corresponding thresholds are also explicitly given. The elastic scattering lengths, effective ranges, and the compositeness coefficients are provided in the last three columns. Since the mass of the $\psi(4260)$ is below the $D_{1} \bar{D}$ threshold, the probabilistic interpretation of $X$ does not hold in this situation [7].

\begin{tabular}{|c|c|c|c|c|c|c|}
\hline Resonance & $\begin{array}{c}\text { Mass } \\
(\mathrm{MeV})\end{array}$ & $\begin{array}{l}\text { Width } \\
(\mathrm{MeV})\end{array}$ & $\begin{array}{c}\text { Threshold } \\
(\mathrm{MeV})\end{array}$ & $\begin{array}{c}a \\
(\mathrm{fm})\end{array}$ & $\begin{array}{c}r \\
(\mathrm{fm})\end{array}$ & $X$ \\
\hline$Z_{c}(3900)$ & $3886.6 \pm 2.4$ & $28.2 \pm 2.6$ & $D \bar{D}^{*}(3875.8)$ & $-0.94 \pm 0.12$ & $-2.40 \pm 0.21$ & $0.49 \pm 0.06$ \\
\hline$X(4020)$ & $4024.1 \pm 1.9$ & $13 \pm 5$ & $D^{*} \bar{D}^{*}(4017.1)$ & $-1.04 \pm 0.26$ & $-3.89 \pm 1.42$ & $0.39 \pm 0.12$ \\
\hline$\psi(4260)$ & $4230 \pm 8$ & $55 \pm 19$ & $D_{1} \bar{D}(4289.2)$ & $-1.04 \pm 0.06$ & $-0.54 \pm 0.03$ & --- \\
\hline$\psi(4660)$ & $4643 \pm 9$ & $72 \pm 11$ & $\Lambda_{c} \bar{\Lambda}_{c}(4572.9)$ & $-0.22 \pm 0.04$ & $-1.98 \pm 0.28$ & $0.24 \pm 0.04$ \\
\hline$\chi_{c 1}(4140)(\mathrm{LHCb})$ & $4146.5 \pm 6.4$ & $83 \pm 30$ & $D_{s} \bar{D}_{s}^{*}(4080.5)$ & $-0.27 \pm 0.06$ & $-1.79 \pm 0.61$ & $0.29 \pm 0.08$ \\
\hline$\chi_{c 1}(4140)$ (ŁHCb) & $4147.1 \pm 2.4$ & $15.7 \pm 6.3$ & $D_{s} \bar{D}_{s}^{*}(4080.5)$ & $-0.06 \pm 0.02$ & $-9.10 \pm 3.86$ & $0.06 \pm 0.02$ \\
\hline$\chi_{c 1}(4140)(\mathrm{PDG})$ & $4146.8 \pm 2.4$ & $22 \pm 8$ & $D_{s} \bar{D}_{s}^{*}(4080.5)$ & $-0.09 \pm 0.03$ & $-6.49 \pm 2.40$ & $0.08 \pm 0.03$ \\
\hline
\end{tabular}

\section{Phenomenological Discussions}

Before entering the detailed discussions, we stress that the theoretical approach developed is based on the elastic $S$-wave two-body scattering. Strictly speaking, the present formalism applies to the scattering of two stable hadrons. A rigorous approach to handle the presence of unstable hadrons in the scattering process is to perform the study within threeor even few-body scattering [26], which is clearly beyond the scope of the present work. Another indirect way to understand the role of the width in the unstable hadrons is to use a complex mass $\left(m_{i} \longrightarrow m_{i}-i \Gamma_{i} / 2\right)$ in the expression for the three-momentum, Eq. (4), which then reads

$$
k_{R}=\sqrt{2 \mu_{m}\left(E_{R}-m_{\mathrm{th}}+i \frac{\Gamma}{2}\right)} .
$$

In this way, one can take into account the self-energy effects of the decaying channels. This is applicable, as compared with a full three-body study, if $\Gamma$ is much smaller than the difference between the mass of the resonance and the threshold of the decay channel, as indicated by the results from [26] concerning the $D \bar{D}^{*}, \bar{D} D^{*}$ and $D \bar{D} \pi$ scattering and the $X(3872)$ resonance. This is the case for the $D_{1}$ hadron, and the numerical results obtained with a complex mass are also indicated below. Another interesting point is that unstable hadrons could introduce additional crossedchannel contributions, comparing with stable ones; see, e.g., [26].

The crossed-channel effects, such as the light-flavor hadron exchanges, are neglected in (1), which is strictly valid in the near-threshold region before any other branch-point singularity, associated with the onset of crossed channels, sets in. Nonetheless, the theoretical formalism presented here can be used also to study the systems in which the crossedchannel dynamics can be treated perturbatively. In $[9,10]$, the resonances $\Lambda_{c}$ (2595), $Z_{b}(10610)$ and $Z_{b}(10650)$ have been successfully addressed within this formalism. In the following, we tentatively generalize the discussions to the
$Z_{c}(3900), X(4020), \chi_{c 1}(4140), \psi(4260)$ and $\psi(4660)$, which may be composed by some specific $S$-wave open-charm twobody states and lie close to their thresholds.

The masses and widths of the $Z_{c}(3900), X(4020)$, $\chi_{c 1}(4140), \psi(4260)$, and $\psi(4660)$ and the thresholds of the nearby two-body states are collected in Table 1 . The spin and parity of the $Z_{c}(3900), \chi_{c 1}(4140), \psi(4260)$, and $\psi(4660)$ given in the PDG [2] are compatible with the $S$-wave elastic scattering of $D \bar{D}^{*}, D_{s} \bar{D}_{s}^{*}, D_{1} \bar{D}$ and $\Lambda_{c} \bar{\Lambda}_{c}$, respectively. For the $X(4020)$, its spin and parity are not confirmed by experiments yet. By assuming the $S$-wave molecular nature of $D^{*} \bar{D}^{*}$, a possible quantum number $J^{P C}$ of the $X(4020)$ would be $1^{+-}$.

For the $Z_{c}(3900)$, we see that standard values of $a$ and $r$, corresponding to the typical scale of the long-range force of strong interactions, result from the $D \bar{D}^{*}$ scattering, according to the results in Table 1 . The component of $D \bar{D}^{*}$ inside the $Z_{c}(3900)$ is as important as the other degrees of freedom (d.o.f), according to the compositeness $X \sim 0.5$. This finding qualitatively agrees with the conclusion from the pole-counting-rule study [27]. We have used the error bars of the masses and widths of the resonances to estimate the uncertainties of the $a, r$, and $X$ and neglected the tiny error bars of the thresholds. For the $X(4020)$, a somewhat large value for $|r|$ is obtained. Both the $D^{*} \bar{D}^{*}$ and other d.o.f play important roles in the formation of $X(4020)$.

It is proposed in $[28,29]$ that the $\psi(4260)$ could be a possible $S$-wave $D_{1} \bar{D}$ resonance. If one assumes that the $S$ wave $D_{1} \bar{D}$ is the only source which is responsible for the resonance pole, the resulting scattering length and effective range can be found in Table 1. Standard values of $a$ and $r$ around $1 \mathrm{fm}$ are obtained. However, because the resonance pole of $\psi(4260)$ is below the $D_{1} \bar{D}$ threshold, we can not use the recipe in (13) for the probabilistic interpretation [7]. Nonetheless, since the presence of a close to threshold CDD pole is characterized by having a small scattering length and a big effective range in magnitudes, the natural values for $a$ and $r$ given in Table 1 favor the interpretation offered in $[28,29]$. 
TABLE 2: Set of numbers $|X|,|\widetilde{X}|[15],|\widehat{X}|[16]$ and $U$ [15-17] for the resonances considered in Table 1. No criteria for the probabilistic interpretation of the compositeness is met in the case of the $\psi(4260)$ resonance.

\begin{tabular}{|c|c|c|c|c|c|}
\hline \multirow[b]{2}{*}{ Resonance } & \multicolumn{5}{|c|}{ Asymptotic } \\
\hline & State & $|X|$ & $\widetilde{X}$ & $\widehat{X}$ & $U$ \\
\hline$Z_{c}(3900)$ & $D \bar{D}^{*}$ & $0.49 \pm 0.06$ & $0.18 \pm 0.02$ & $0.30 \pm 0.02$ & $0.60 \pm 0.10$ \\
\hline$X(4020)$ & $D^{*} \bar{D}^{*}$ & $0.39 \pm 0.12$ & $0.16 \pm 0.04$ & $0.27 \pm 0.06$ & $0.47 \pm 0.19$ \\
\hline$\psi(4260)$ & $D_{1} \bar{D}$ & $4.5 \pm 1.6$ & $0.45 \pm 0.02$ & $0.49 \pm 0.01$ & $8.1 \pm 3.2$ \\
\hline$\psi(4660)$ & $\Lambda_{c} \bar{\Lambda}_{c}$ & $0.24 \pm 0.04$ & $0.11 \pm 0.02$ & $0.19 \pm 0.03$ & $0.27 \pm 0.05$ \\
\hline$\chi_{c 1}(4140)(\mathrm{LHCb})$ & $D_{s} \bar{D}_{s}^{*}$ & $0.29 \pm 0.08$ & $0.12 \pm 0.03$ & $0.22 \pm 0.05$ & $0.33 \pm 0.12$ \\
\hline$\chi_{c 1}(4140)$ (ЕHCb) & $D_{s} \bar{D}_{s}^{*}$ & $0.06 \pm 0.02$ & $0.03 \pm 0.01$ & $0.06 \pm 0.02$ & $0.06 \pm 0.02$ \\
\hline$\chi_{c 1}(4140)(\mathrm{PDG})$ & $D_{s} \bar{D}_{s}^{*}$ & $0.08 \pm 0.03$ & $0.04 \pm 0.01$ & $0.08 \pm 0.03$ & $0.09 \pm 0.03$ \\
\hline
\end{tabular}

As shown in [10] when the position of the CDD pole tends to threshold, $M_{\mathrm{CDD}} \longrightarrow m_{\mathrm{th}}$, the resulting $a$ and $r$ tend to

$$
\begin{aligned}
& a \longrightarrow \frac{M_{\mathrm{CDD}}-m_{\mathrm{th}}}{\lambda}, \\
& r \longrightarrow-\frac{\lambda}{\mu_{m}\left(M_{\mathrm{CDD}}-m_{\mathrm{th}}\right)^{2}},
\end{aligned}
$$

where $\lambda$ is the residue of the CDD pole. The bigger this residue is, the sooner this behavior sets in. When the finite width of the $D_{1}$ is included via (14), the scattering length $a$ and effective range $r$ are shifted to $-1.10 \pm 0.07 \mathrm{fm}$ and $-0.55 \pm 0.04 \mathrm{fm}$, respectively, which are compatible with the results shown in Table 1 within uncertainties. Therefore the effects from the finite width of the $D_{1}$ are indeed small. We also note that in [30], the large coupling of $\psi(4260)$ to $\omega \chi_{c 0}$ is pointed out. Employing this coupling [7] obtained that $X_{\omega \chi_{c 0}} \sim 0.2$.

Due to the closeness of the $\psi(4660)$ to the $\Lambda_{c} \bar{\Lambda}_{c}$ threshold and the compatibility of its quantum numbers with the $S$ wave $\Lambda_{c} \bar{\Lambda}_{c}$, we also explore the possibility that the elastic $S$-wave $\Lambda_{c} \bar{\Lambda}_{c}$ scattering is responsible for the $\psi(4660)$ pole. However, the small value of compositeness coefficient $X$ in Table 1 indicates that the $\Lambda_{c} \bar{\Lambda}_{c}$ component plays a minor role and other d.o.f plays a more important role inside the $\psi(4660)$.

The quantum numbers of the $S$-wave $D_{s} \bar{D}_{s}^{*}$ scattering is compatible with the preferred $J^{P C}=1^{++}$of the $\chi_{c 1}(4140)$ [2]. Notice that the new determinations of the masses and widths from $\mathrm{LHCb}[31,32]$ are obviously larger than the previous measurements. We explicitly give different values for the masses and widths of $\chi_{c 1}(4140)$ in Table 1 . In all cases, it seems that the $D_{s} \bar{D}_{s}^{*}$ component plays a minor role inside $\chi_{c 1}(4140)$.

We have employed the approach of $[7,10]$ which conclude a probabilistic interpretation of $|X|$ for $M_{R}>m_{\mathrm{th}}$. As explained in more detail in $[7,8]$, this result is based on a phase redefinition of the resonance couplings (whose phases are affected by the non-resonant terms around the pole [33]), and on a direct observation of the resonance-peak signal stemming from its Laurent series for physical values of the energy. In contrast, [15-17] propose some ad-hoc manipulations on the complex numbers $X$ and $Z=1-X$ so as to end with positive definite values between 0 and 1 . We compare our results with theirs below.

References $[15,17]$ construct from $|X|$ and $|Z|$ other numbers $\widetilde{X}, \widetilde{Z}$ and $U$, which are defined as

$$
\begin{aligned}
& \widetilde{X}=\frac{1}{2}+\frac{|X|-|1-X|}{2}, \\
& \widetilde{Z}=\frac{1}{2}+\frac{|1-X|-|X|}{2}, \\
& U=|X|+|1-X|-1 .
\end{aligned}
$$

By construction they fulfill that $\widetilde{X}+\widetilde{Z}=1$ and $0 \leq \widetilde{X}, \widetilde{Z} \leq 1$. The parameter $U$ measures the degree of cancellation between the complex numbers $X$ and $Z$, whose sum is 1 . By geometrical reasoning, see Figure 1 of [15], $\pm U / 2$ is interpreted as the uncertainty in the probabilistic interpretation of $\widetilde{X}$.

In turn, [16] introduces other numbers $\widehat{X}$ and $\widehat{Z}$ defined by

$$
\begin{aligned}
& \widehat{X}=\frac{|X|}{1+U}, \\
& \widehat{Z}=\frac{|1-X|}{1+U} .
\end{aligned}
$$

These numbers also fulfill by construction that $0 \leq \widehat{X}, \widehat{Z} \leq 1$ and $\widehat{X}+\widehat{Z}=1$. This reference claims that $\widehat{X}$ and $\widehat{Z}$ have a probabilistic interpretation in connection with the weight of the different continuum states in a resonance if $U \ll 1$. It is also noticed that the difference between $\widetilde{X}$ and $\widehat{X}$ tends to zero linearly in $U$ for $U \longrightarrow 0$.

We give in Table 2 the values of $|X|, \widetilde{X}, \widehat{X}$ and $U$ for the resonances shown in Table 1. For the last two entries of the $\chi_{c 1}(4140)$ one has that $U \ll 1$ and the same quantitative conclusion on the very small size of the compositeness of $D_{s} \bar{D}_{s}^{*}$ results from all these numbers. Still small values for $U \lesssim 0.3$ results for the first entry of the $\chi_{c 1}(4140)$ and for the $\psi(4660)$, and a similar conclusion on the relatively small size of the weight of the two-body continuum states results from all the instances. Notice that it is always the case in these examples that $|X| \simeq \widehat{X}$, while $\widetilde{X}$ is different by around a factor of 2 . This is because of the following [15]:

$$
|| X|-\widetilde{X}|=\frac{U}{2}
$$


as it is clear from (16), and $U$ is as large as $|X|$ in these cases. Thus, the approach that we follow here, based on $[7,10]$ and summarized above, favors the use of $\widehat{X}$ of [16] over $\widetilde{X}$ of [15].

For the resonance $X(4020)$ one has that $U \approx 0.5$. Now, the difference between the central values of $|X|$ and $\widehat{X}$ is larger, although they drive to a similar conclusion on the weight of the continuum state. The value of $U$ is larger for the $Z_{c}(3900)$, with $U / 2 \approx 0.3$, and the uncertainty $U / 2$ in the interpretation of the numbers $\widehat{X}$ and $\widetilde{X}$, as argued in [15], becomes very important, driving to values that differ between each other by around a factor of 2 . Finally, any of the criteria for the probabilistic interpretation of the compositeness of the $\psi(4260)$ cannot be applied since now $U$ is huge.

Let us also mention that the ERE for scattering up to and including the effective range, like in our study here, drives necessarily to purely imaginary values for $X$, since $X=$ $i \gamma_{k}^{2}=-i k_{i} / k_{r}$. This invalidates the interpretation of $\operatorname{Re} X$ as the compositeness for the case of a resonance, as advocated in [24], because it is always equal to zero in our case, no matter the nature of the resonance under study.

\section{Summary and Conclusions}

In this work, we have combined the effective range expansion, unitarity, analyticity, and the compositeness coefficient to study the resonance dynamics around the threshold energy region. We only focus on the elastic $S$-wave scattering throughout. In our formalism, the scattering length, effective range, and the compositeness coefficient can be straightforwardly calculated, if the mass and width of the resonance are provided.

We have applied the theoretical formalism to the $Z_{c}(3900), X(4020), \chi_{c 1}(4140), \psi(4260)$ and $\psi(4660)$. The resonance poles are assumed to be generated by the elastic $S$-wave scattering of $D \bar{D}^{*}, D^{*} \bar{D}^{*}, D_{s} \bar{D}_{s}^{*}, D_{1} \bar{D}$ and $\Lambda_{c} \bar{\Lambda}_{c}$, respectively. According to the values in Table 1, we tentatively conclude that both the $D \bar{D}^{*}$ and other degrees of freedom are equally important inside the $Z_{c}(3900)$. The $D^{*} \bar{D}^{*}$ component inside the $X(4020)$ is also important, while, for the $\chi_{c 1}(4140)$ and $\psi(4660)$, the $D_{s} \bar{D}_{s}^{*}$ and $\Lambda_{c} \bar{\Lambda}_{c}$ components seem playing minor roles, respectively. In addition, the natural values for $a \simeq-1 \mathrm{fm}$ and $r \simeq-0.5 \mathrm{fm}$ in the case of the $\psi(4260)$ are compatible with its interpretation in $[28,29]$ as a $D_{1} \bar{D}$ molecular state.

\section{Data Availability}

The data used to support the findings of this study are included within the article.

\section{Conflicts of Interest}

The authors declare that they have no conflicts of interest.

\section{Acknowledgments}

Zhi-Hui Guo would like to thank En Wang for the informative communication on the $\chi_{c 1}(4140)$. This work is funded in part by the NSFC under Grants nos. 11575052 and 11805012, the Natural Science Foundation of Hebei Province under Contract no. A2015205205, and the MINECO (Spain) and FEDER (EU) Grant FPA2016-77313-P.

\section{References}

[1] S. K. Choi, [Belle Collaboration] et al., "Observation of a Narrow Charmoniumlike State in Exclusive $\mathrm{B}^{ \pm} \longrightarrow \mathrm{K}^{ \pm} \pi^{+} \pi^{-} \mathrm{J} / \psi$ Decays," Physical Review Letters, vol. 91, Article ID 262001, 2003.

[2] M. Tanabashi, K. Hagiwara, and K. Hikasa, "Review of particle physics," Physical Review D: Particles, Fields, Gravitation and Cosmology, vol. 98, no. 3, Article ID 030001, 2018.

[3] H. X. Chen, W. Chen, X. Liu, and S. L. Zhu, "The hidden-charm pentaquark and tetraquark states," Physics Reports, vol. 639, pp. $1-121,2016$.

[4] F. K. Guo, C. Hanhart, U. G. Meißner, Q. Wang, Q. Zhao, and B. S. Zou, "Hadronic molecules," Reviews of Modern Physics, vol. 90, no. 1, Article ID 015004, 2018.

[5] H. A. Bethe, "Theory of the effective range in nuclear scattering," Physical Review Journals, vol. 76, pp. 38-50, 1949.

[6] M. A. Preston and B. K. Bhaduri, Structure of the Nucleus, Addison-Wesley Publishing Company, Inc., Massachusetts, Mass, USA, 1975.

[7] Z. H. Guo and J. A. Oller, "Probabilistic interpretation of compositeness relation for resonances," Physical Review D, vol. 93, no. 9, Article ID 096001, 2016.

[8] J. A. Oller, "New results from a number operator interpretation of the compositeness of bound and resonant states," Annals of Physics, vol. 396, pp. 429-458, 2018.

[9] Z. H. Guo and J. A. Oller, "Resonance on top of thresholds: the $\Lambda_{c}(2595)^{+}$as an extremely fine-tuned state," Physical Review D, vol. 93, no. 5, Article ID 054014, 2016.

[10] X. W. Kang, Z. H. Guo, and J. A. Oller, "General considerations on the nature of $Z_{b}(10610)$ and $Z_{b}(10650)$ from their pole positions," Physical Review D: Particles, Fields, Gravitation and Cosmology, vol. 94, no. 1, Article ID 014012, 2016.

[11] Z. H. Guo and J. A. Oller, "Anatomy of the newly observed hidden-charm pentaquark states: $P_{c}(4312), P_{c}(4440)$ and $P_{c}$ (4457)," Physics Letters B, vol. 793, pp. 144-149, 2019.

[12] L. Y. Dai, J. Haidenbauer, and U. G. Meißner, "Re-examining the $\mathrm{X}(4630)$ resonance in the reaction $e^{+} e^{-} \longrightarrow \Lambda_{c}^{+} \bar{\Lambda}_{c}^{-}$," Physical Review D, vol. 96, no. 11, Article ID 116001, 2017.

[13] G. Cotugno, R. Faccini, A. D. Polosa, and C. Sabelli, "Charmed baryonium," Physical Review Letters, vol. 104, no. 13, Article ID 132005, 2010.

[14] F. Guo, J. Haidenbauer, C. Hanhart, and U. Meißner, "Reconciling the $X(4630)$ with the $Y(4660)$," Physical Review D: Particles, Fields, Gravitation and Cosmology, vol. 82, no. 9, Article ID 094008, 2010.

[15] Y. Kamiya and T. Hyodo, "Generalized weak-binding relations of compositeness in effective field theory," PTEP: Progress of Theoretical and Experimental Physics, vol. 2017, no. 2, Article ID 023D02, 2017.

[16] T. Sekihara, T. Arai, J. Yamagata-Sekihara, and S. Yasui, "Compositeness of baryonic resonances: Application to the $\Delta(1232), N(1535)$, and N(1650) resonances," Physical Review C: Nuclear Physics, vol. 93, no. 3, Article ID 035204, 2016.

[17] Y. Kamiya, T. Hyodo, and C. Phys. Rev, "Structure of nearthreshold quasibound states," Physical Review C, vol. 93, no. 3, Article ID 035203, 2016. 
[18] X. Kang and J. A. Oller, "Different pole structures in line shapes of the X(3872)," The European Physical Journal C, vol. 77, no. 6, article 399, 2017.

[19] V. Baru, J. Haidenbauer, C. Hanhart, Y. Kalashnikova, and A. E. Kudryavtsev, "Evidence that the a $(0)(980)$ and $\mathrm{f}(0)(980)$ are not elementary particles," Physics Letters B, vol. 586, no. 1-2, pp. 53-61, 2004.

[20] C. Hanhart, Y. S. Kalashnikova, and A. V. Nefediev, "Interplay of quark and meson degrees of freedom in a near-threshold resonance: multi-channel case," The European Physical Journal A, vol. 47, article 101, 2011.

[21] T. Hyodo, D. Jido, and A. Hosaka, "Compositeness of dynamically generated states in a chiral unitary approach," Physical Review C: Nuclear Physics, vol. 85, no. 1, Article ID 015201, 2012.

[22] F. Aceti and E. Oset, "Wave functions of composite hadron states and relationship to couplings of scattering amplitudes for general partial waves," Physical Review D: Particles, Fields, Gravitation and Cosmology, vol. 86, no. 1, Article ID 014012, 2012.

[23] T. Sekihara, T. Hyodo, and D. Jido, "Comprehensive analysis of the wave function of a hadronic resonance and its compositeness," PTEP: Progress of Theoretical and Experimental Physics, vol. 2015, no. 6, Article ID 063D04, 2015.

[24] F.-H. Liu, T. Tian, H. Zhao, and B.-C. Li, "Meson-baryon components in the states of the baryon decuplet," The European Physical Journal A, vol. 50, article 57, 2014.

[25] S. Weinberg, "Elementary particle theory of composite particles," Physical Review A: Atomic, Molecular and Optical Physics, vol. 130, no. 2, pp. 776-783, 1963.

[26] V. Baru, A. A. Filin, C. Hanhart, Y. S. Kalashnikova, A. E. Kudryavtsev, and A. V. Nefediev, "Three-body $D \bar{D} \pi$ dynamics for the X(3872)," Physical Review D: Particles, Fields, Gravitation and Cosmology, vol. 84, no. 7, Article ID 074029, 2011.

[27] Q. Gong, Z. Guo, C. Meng, G. Tang, Y. Wang, and H. Zheng, " $Z_{c}(3900)$ as a $\bar{D} D^{*}$ molecule from the pole counting rule," Physical Review D: Particles, Fields, Gravitation and Cosmology, vol. 94, no. 11, Article ID 114019, 2016.

[28] Q. Wang, C. Hanhart, and Q. Zhao, "Decoding the Riddle of $Y(4260)$ and $Z_{c}(3900)$," Physical Review Letters, vol. 111, no. 13, Article ID 132003, 2013.

[29] Q. Wang, M. Cleven, F. Guo et al., “Y(4260): Hadronic molecule versus hadro-charmonium interpretation," Physical Review D: Particles, Fields, Gravitation and Cosmology, vol. 89, no. 3, Article ID 034001, 2014.

[30] L. Dai, M. Shi, G. Tang, and H. Zheng, "Nature of X(4260)," Physical Review D: Particles, Fields, Gravitation and Cosmology, vol. 92, no. 1, Article ID 014020, 2015.

[31] R. Aaij, B. Adeva, and M. Adinolfi, "Publisher's Note: First Experimental Study of Photon Polarization in Radiative $B_{s}^{0}$ Decays," Physical Review Letters, vol. 118, no. 2, Article ID 022003, 2017.

[32] R. Aaij et al., "Amplitude analysis of $B^{+} \longrightarrow J / \psi \phi K^{+}$decays," Physical Review D: Particles, Fields, Gravitation and Cosmology, vol. 95, no. 1, Article ID 012002, 2017.

[33] S. Weinberg, The Quantum Field Theory of Fields. Volume I Foundations, Cambridge University Press, New York, NY, USA, 1995. 

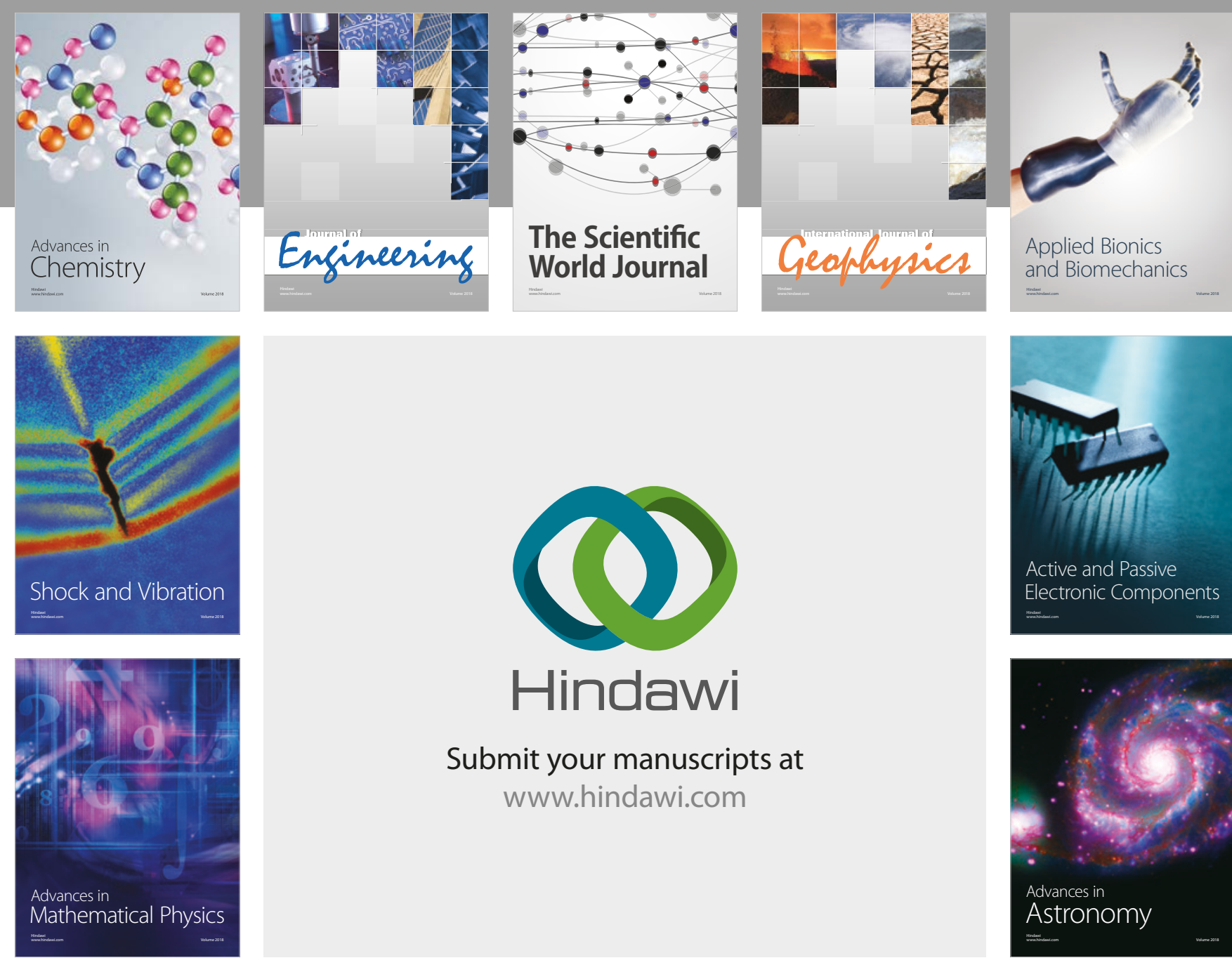

Submit your manuscripts at

www.hindawi.com

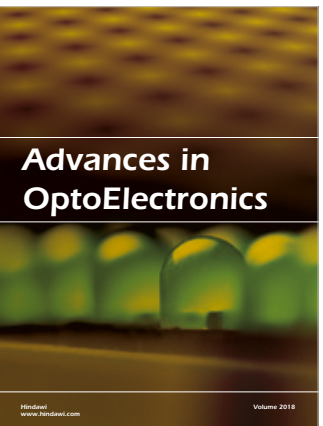

\section{Rotcting Machinery}
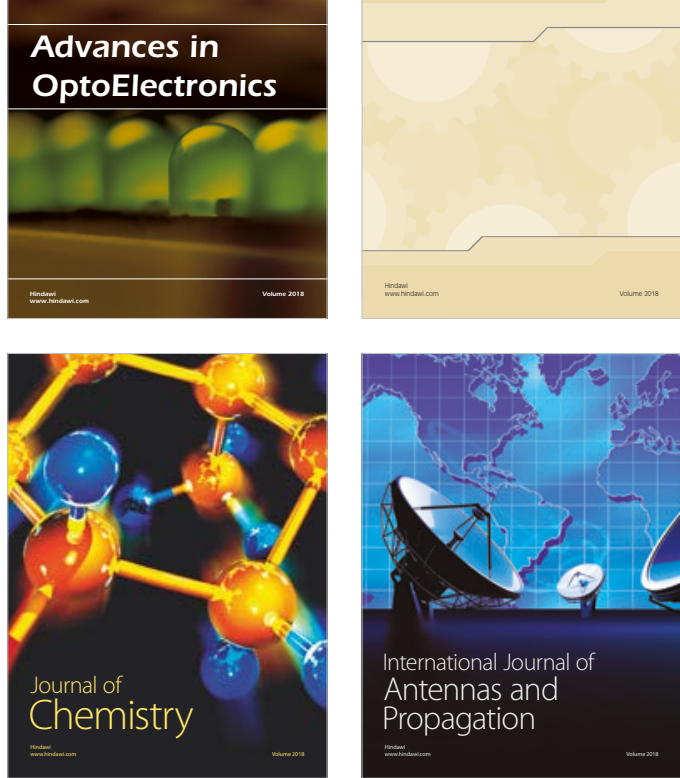

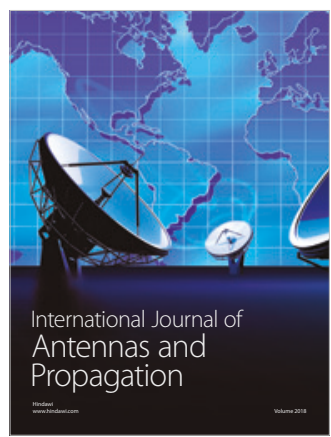

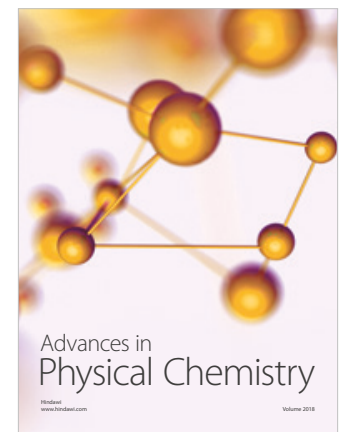

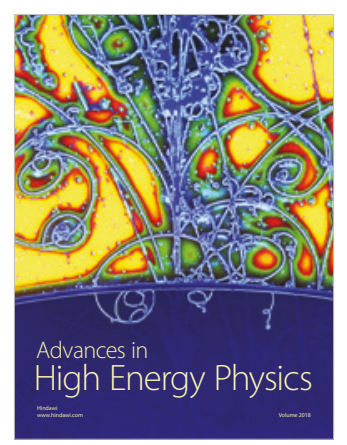

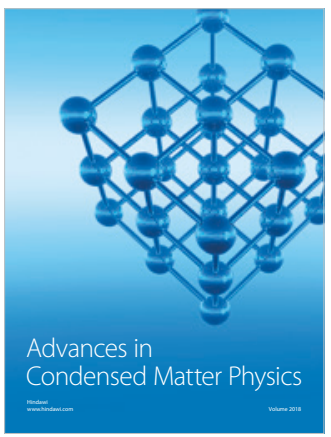

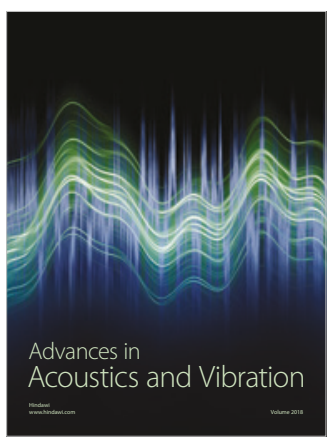

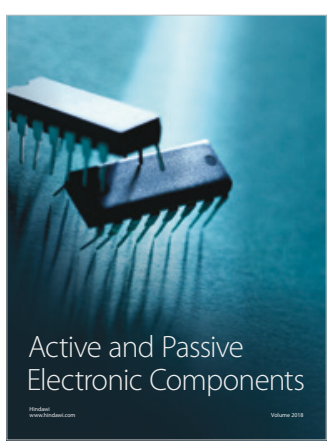
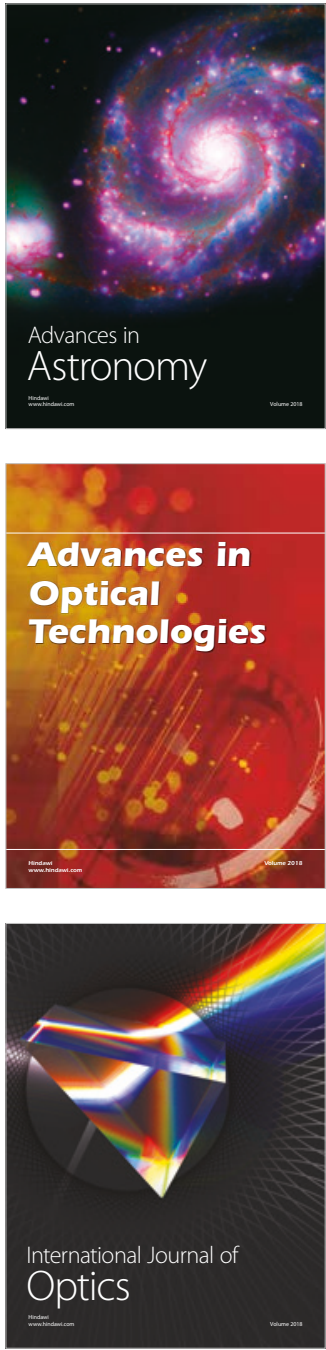